\title{
ORIGINAL ARTICLE \\ Changes in renal function during acute spinal cord injury: implications for pharmacotherapy
}

\author{
V Rodríguez-Romero ${ }^{1}$, L Cruz-Antonio ${ }^{2}$, RE Franco-Bourland ${ }^{3,4}$, G Guízar-Sahagún ${ }^{4,5}$ \\ and G Castañeda-Hernández ${ }^{1}$
}

Study design: Laboratory investigation in rats submitted to experimental spinal cord injury (SCI).

Objective: To characterize changes in renal function during acute SCI.

Methods: Sprague Dawley rats were subjected to severe spinal cord contusion at T8 level or to laminectomy as control. Twenty-four hours after spine surgery, clearance assessments of a single dose of iohexol $\left(120 \mathrm{mg} \mathrm{kg}^{-1}\right)$ or of $p$-aminohippuric acid (PAH, $100 \mathrm{mg} \mathrm{kg}^{-1}$ ) were used to evaluate glomerular filtration rate (GFR) and tubular secretion (TS), respectively. Blood sampling was used to determine concentrations of both compounds by high-performance liquid chromatography for pharmacokinetic measurements. Results: lohexol clearance decreased significantly after injury, which resulted in increased concentrations and half-life of iohexol in blood; PAH clearance remained unchanged.

Conclusion: GFR but not TS is altered during spinal shock. These observations should be of interest to professionals caring for early cord-injured patients, in order to prevent toxicity and therapeutic failure when administering drugs eliminated by the kidney.

Spinal Cord (2013) 51, 528-531; doi:10.1038/sc.2013.35; published online 23 April 2013

\section{Keywords: glomerular filtration rate; iohexol; renal dysfunction; tubular secretion}

\section{INTRODUCTION}

Spinal cord injury (SCI) causes numerous systemic and metabolic alterations that can interfere with the efficacy and security of drug use. ${ }^{1}$ Changes in drug disposition and kinetics have been documented in patients and animal models of SCI as a consequence of autonomic nervous system dysfunction. ${ }^{2-4}$ Precise understanding of the pathophysiological mechanisms involved in pharmacokinetic and pharmacodynamic alterations associated with $\mathrm{SCI}$ is required to optimize pharmacological treatments.

The kidney is the major regulator of internal fluid environment; therefore, physiologic changes associated with renal dysfunction can have pronounced effects on drug performance, particularly of drugs eliminated by the kidney such as aminoglycosides and quinolones, which are widely used after SCI.

There are reports that show decrease of glomerular filtration rate (GFR) in patients with chronic cord injury. ${ }^{5,6}$ However, information on GFR during early stages of SCI is scarce and wrongly based on creatinine measurements, ${ }^{7}$ which are faulty because they are affected by muscle mass catabolism occurring at this stage of SCI.

Our objective here was to assess renal function in the acute stage of experimental SCI by determining (1) GFR from clearance of an exogenous compound unrelated to endogenous compounds derived from muscle catabolism, and (2) tubular secretion (TS). We have hypothesized that during spinal shock produced after experimental SCI, renal function is altered showing decreased GFR and increased TS as a compensatory mechanism.

\begin{abstract}
MATERIALS AND METHODS
Experimental design

Our local institutional Animal Care Committee approved this study. Animals were handled according to the ethical guidelines of our institution and $\mathrm{NIH}$ standards. Assessment of GFR and TS was carried out 1 day after spine surgery in groups $(n=5)$ of female Sprague Dawley rats weighing 230-250 g body weight that were randomly assigned to one of the following procedures: SC contusion at T8 level or laminectomy as sham-injury control. Animals were allowed to eat and drink ad libitum and were kept in individual cages.
\end{abstract}

\section{Spinal cord injury and postoperative care}

For spinal surgery, animals were anesthetized with a mixture of ketamine $\left(100 \mathrm{mg} \mathrm{kg}^{-1}\right)$ and xylazine $\left(20 \mathrm{mg} \mathrm{kg}^{-1}\right)$ given intramuscularly. Following laminectomy, a severe SC contusion was inflicted by dropping onto the exposed dura a stainless steel rod weighing $10 \mathrm{~g}$ from a height of $50 \mathrm{~mm}$ using the well-characterized New York University weight-drop impactor device. After cord injury, neurogenic bowel and bladder were handled by manual pressure. Sham-injured controls were only laminectomized. At the end of the study, rats were killed in a $\mathrm{CO}_{2}$ chamber.

\section{Assessment of glomerular filtration rate}

Twenty-four hours after spine surgery, animals (cord- and sham-injured rats) received a single dose of iohexol $\left(120 \mathrm{mg} \mathrm{kg}^{-1}\right)$ intravenously. Blood samples of $100 \mu \mathrm{l}$ were drawn through a catheter inserted in the caudal artery under light ether anesthesia ${ }^{4}$ at the following time points: $0,5,10,15,20,30,45,60$, $90,120,180,240$, and $300 \mathrm{~min}$. The volume of blood extracted was replaced immediately after sampling with the same volume of physiological isotonic

${ }^{1}$ Departamento de Farmacología, Centro de Investigación y de Estudios Avanzados del Instituto Politécnico Nacional, México City, México; ${ }^{2}$ Facultad de Estudios Superiores Zaragoza, Universidad Nacional Autónoma de México, México City, México; ${ }^{3}$ Servicio de Bioquímica, Instituto Nacional de Rehabilitación, México City, México; ${ }^{4}$ Proyecto Camina A.C., México City, México and 5 Unidad de Investigación en Enfermedades Neurológicas, Instituto Mexicano del Seguro Social, México City, México

Correspondence: Dr L Cruz-Antonio, Área Farmacéutica, Carrera de QFB, Facultad de Estudios Superiores Zaragoza de la Universidad Nacional Autónoma de México, Av. Guelatao 66, Col. Ejército de Oriente, 09230 México City, DF, México.

E-mail: letycruza@yahoo.com.mx

Received 17 December 2012; revised 26 February 2013; accepted 16 March 2013; published online 23 April 2013 
saline to avoid reduction in the circulating volume. Iohexol concentration in whole blood was determined by high-performance liquid chromatography, as previously described, ${ }^{8}$ with minor modifications. Briefly, $50 \mu \mathrm{l}$ sulfapyridine $\left(1 \mathrm{mg} \mathrm{ml}^{-1}\right)$ were added to blood samples as an internal standard, and then precipitated with $0.850 \mathrm{ml}$ of $5 \%$ perchloric acid. Samples were shaken in a vortex for $1 \mathrm{~min}$ and then centrifuged for $10 \mathrm{~min}$ at $3000 \times g$. Supernatants were analyzed by high-performance liquid chromatography at room temperature using a Nova-Pak C18 column (Waters Corp., Milford, MA, USA). The mobile phase consisted of a solution of methanol-water $6: 94 \mathrm{v} / \mathrm{v}$, at a constant flow rate of $2.0 \mathrm{ml} \mathrm{min}^{-1}$; the effluent was monitored by UV detection at $254 \mathrm{~nm}$. Retention times for iohexol and the internal standard were 1.75 and $4.38 \mathrm{~min}$, respectively. Individual curves of blood iohexol concentrations against time were plotted and analysis of pharmacokinetic parameters (half-life, area under the curve, volume distribution and clearance) was performed using a noncompartmental approach, as previously described. ${ }^{3,4}$

\section{Assessment of tubular secretion}

Also $24 \mathrm{~h}$ after spine surgery, other groups of cord- and sham-injured rats received a single $100 \mathrm{mg} \mathrm{kg}^{-1}$ intravenous dose of $p$-aminohippuric acid (PAH) to assess tubular secretory function. Blood samples of $200 \mu \mathrm{l}$ were obtained as previously described ${ }^{4}$ at the following time points: $0,2.5,5,10,15$, 30,60 , and $120 \mathrm{~min}$, and circulating volume was immediately restored after blood sampling. PAH concentration in plasma was determined by highperformance liquid chromatography, as previously described, ${ }^{9}$ with minor modifications. Briefly, after liquid-liquid extraction with methanol, plasma samples were analyzed on a Nova-Pak C18 column (Waters Corp.,) using a solution of $0.1 \mathrm{M}$ acetic acid-acetonitrile $(99: 1, \mathrm{v} / \mathrm{v}) \mathrm{pH} 3-4$ as mobile phase with UV detection at $285 \mathrm{~nm}$. The flow rate was set at $1 \mathrm{mlmin}^{-1}$. $P$-aminobenzoic acid $\left(50 \mu \mathrm{l}\right.$ of a $250 \mu \mathrm{g} / \mathrm{ml}^{-1}$ solution) was used as internal standard. Retention times for PAH and the internal standard were 2.56 and $4.18 \mathrm{~min}$, respectively. Individual curves of blood PAH concentrations against time were plotted and pharmacokinetic analysis was performed as above.

\section{Statistics}

Comparisons of pharmacokinetic parameters between SCI and control animals were performed using Student's $t$ test. Differences were considered significant when $P<0.05$.

\section{RESULTS}

Animals subjected to SCI showed complete flaccid rear paralysis $24 \mathrm{~h}$ after injury, while animals, which had only been laminectomized exhibited normal locomotion.

Blood levels of iohexol decreased rapidly in sham-injured rats and were undetectable after $120 \mathrm{~min}$; blood concentration of iohexol in rats with cord injury remained higher throughout the study compared with controls and was detectable up to the end of the screening (Figure 1). Except for the volume of distribution, the pharmacokinetic parameters studied for iohexol were altered significantly in paraplegic rats compared with controls (Table 1): systemic clearance decreased to $29 \%$, while half-life and area under the curve increased close to fivefold.

Unexpectedly, blood levels of PAH (Figure 2) and their pharmacokinetic parameters (Table 2) in injured rats were similar to those obtained for controls.

\section{DISCUSSION}

Here we investigated the effect of early SCI on renal function. We present evidence of renal dysfunction during spinal shock. Two separate functions of the nephron were assessed: GFR, as measured by iohexol clearance, and TS, as measured by PAH clearance.

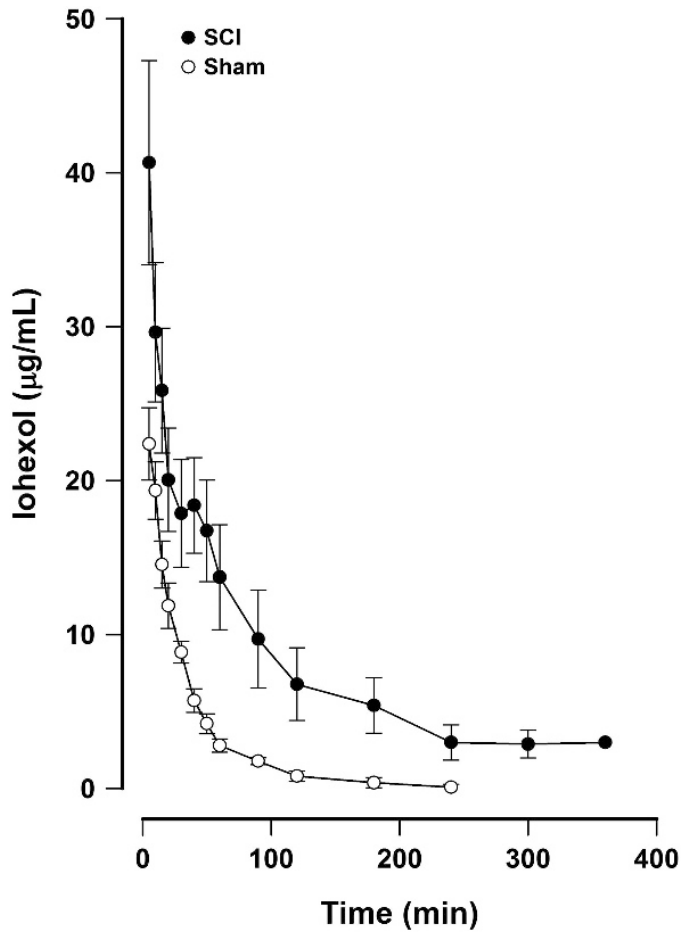

Figure 1 lohexol blood concentration vs time plots after intravenous administration in cord-injured and laminectomized-only control (sham) rats. Data is plotted as mean \pm s.e.m.

Table 1 Pharmacokinetic parameters for iohexol after intravenous administration

\begin{tabular}{lcc}
\hline Group & Sham injury & $S C l$ \\
\hline$t_{1 / 2}(\mathrm{~min})$ & $19.69 \pm 3.94$ & $100.39 \pm 20.68^{\mathrm{a}}$ \\
$\left.\mathrm{AUClnf}(\mu \mathrm{gh} \mathrm{ml})^{-1}\right)$ & $11.83 \pm 1.63$ & $62.33 \pm 15.49^{\mathrm{a}}$ \\
$\mathrm{Vd}\left(\mathrm{l} \mathrm{kg}{ }^{-1}\right)$ & $0.97 \pm 0.36$ & $0.56 \pm 0.14$ \\
$\mathrm{Cl}\left(\mathrm{ml} \mathrm{min}^{-1}\right)$ & $4.01 \pm 0.41$ & $1.15 \pm 0.39^{\mathrm{a}}$
\end{tabular}

Abbreviations: AUCInf, area under the curve extrapolated to infinity; $\mathrm{Cl}$, clearance; $\mathrm{SCl}$, spinal cord injury; $t_{1 / 2}$, half-life; Vd, volume of distribution.

Data are expressed as mean \pm s.e.m.

a $P<0.05$ as determined by Student's $t$ test.

\section{Reduced glomerular filtration rate}

Considering that iohexol undergoes renal clearance by glomerular filtration, ${ }^{9}$ the changes we observed in its blood kinetic parameters reveal that acute SCI significantly decreases GFR, probably as a consequence of renal hypoperfusion.

Renal hypoperfusion during acute SCI can be explained as follows. It is known that acute SCI generates an imbalance in the functions regulated by the autonomic nervous system, mainly due to the lack of sympathetic brain control. ${ }^{1}$ Various neurohumoral counterregulatory systems act in concert to maintain the perfusion of vital organs such as brain and heart. Redistribution of blood volume through constriction of selective vascular beds results in decreased blood perfusion of less critical organs such as kidneys. ${ }^{10,11} \mathrm{We}^{10,12}$ and others $^{13,14}$ have observed that modest arterial hypotension, supraventricular arrhythmias (tachycardia or bradycardia) and relative hypovolemia (due to the pooling of venous blood in the rear limbs and abdominal viscera without changes in central venous pressure) occur during the spinal shock stage after injuries at lower 


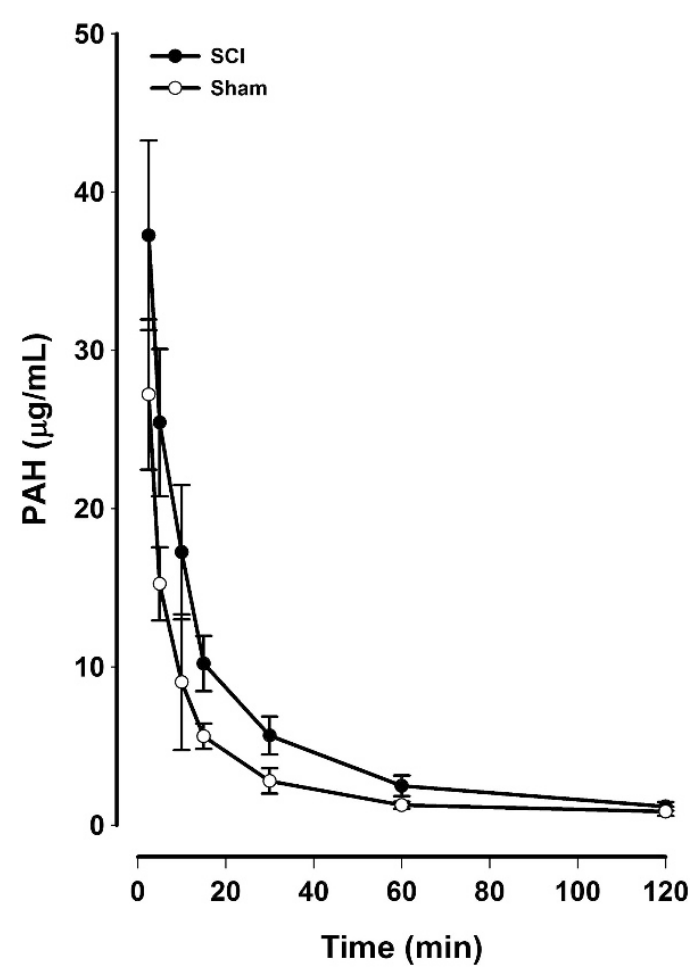

Figure $2 \mathrm{PAH}$ blood concentration vs time plots after intravenous administration in cord-injured and laminectomized-only control (sham) rats. Data is plotted as mean \pm s.e.m.

Table 2 Pharmacokinetic parameters for PAH after intravenous administration

\begin{tabular}{|c|c|c|}
\hline Group & Sham injury & $\mathrm{SCl}$ \\
\hline$t_{1 / 2}(\min )$ & $32.78 \pm 3.89$ & $45.05 \pm 7.70$ \\
\hline AUCInf $\left(\mu \mathrm{gh} \mathrm{ml}^{-1}\right)$ & $9.03 \pm 1.09$ & $11.02 \pm 1.54$ \\
\hline $\operatorname{Vd}\left(\mathrm{kg}^{-1}\right)$ & $9.17 \pm 1.33$ & $8.44 \pm 0.97$ \\
\hline $\mathrm{Cl}\left(\mathrm{ml} \mathrm{min}^{-1}\right)$ & $49.06 \pm 4.96$ & $35.70 \pm 6.20$ \\
\hline
\end{tabular}

Abbreviations: AUCInf, area under the curve extrapolated to infinity; $\mathrm{Cl}$, clearance; $\mathrm{SCl}$, spinal cord injury; $t_{1 / 2}$, half-life; $V d$, volume of distribution. Data are expressed as mean \pm s.e.m.

thoracic levels. It is reasonable to speculate that such cardiovascular alterations could lead to hemodynamic instability, which might contribute to the reduced GFR we have described here. Alternatively, early renal dysfunction has also been related to the systemic inflammatory response produced after acute SCI. ${ }^{15}$

Although there are several methods for measuring GFR, here we chose to use iohexol clearance to examine the impact of acute SCI on GFR for several reasons: (1) creatinine clearance, the clinical standard for GFR measurements, is not an option because creatinine production is significantly altered after SCI due to muscle catabolism by denervation; ${ }^{16,17}$ (2) we are aware that GFR is ideally measured by inulin clearance, however, this method is clinically impractical and costly; $^{16}$ (3) GFR has been measured in patients with SCI using radioactive tracers that correlate closely with inulin clearance; ${ }^{6}$ however, radioisotopic methods have the disadvantage of handling and disposal of radioactive materials and their cost; ${ }^{17}$ (4) no endogenous ideal substance exists for measuring GFR after SCI.

Therefore, GFR measurements require the infusion of an exogenous agent such as iohexol. It is a nonionic, water-soluble compound used clinically as radiocontrast agent. ${ }^{18}$ It is not metabolized, it is eliminated almost entirely by glomerular filtration, and is not secreted or reabsorbed in tubules. It has a low molecular weight and binds poorly to protein. ${ }^{19}$

Considering that iohexol clearance is comparable to that of inulin, ${ }^{19,20}$ we chose this compound for our GFR measurements; in fact, our results (control group) are in agreement with those of Sturgeon $^{21}$ for inulin clearance in normal Sprague Dawley rats. Furthermore, this technique has proven to be reproducible, simple and fast. ${ }^{20}$

\section{Preserved tubular secretion}

In view of the fact that acute SCI does not modify kinetics of PAH clearance, suggesting that active TS is not altered during spinal shock, changes in iohexol clearance can confidently be attributed solely to alterations in GFR. Also, it appears that the pathophysiological mechanisms involved in GFR dysfunction have no effect on TS, in particular those related with renal ischemia.

By determining PAH clearance, we assessed the organic anion transporting mechanism, ${ }^{22}$ kidney's major secretory pathway. PAH is secreted in renal tubules from renal vessels; only $20-30 \%$ is filtered by the glomerulus and is not reabsorbed by tubules. It has been shown that PAH concentration profile along the length of the proximal convolution does not change markedly with variations in GFR. ${ }^{23}$ The fraction of PAH that passes the glomerulus and enters tubular cells of the nephron (via peritubular capillaries) is completely secreted. ${ }^{9}$

\section{Consequences of renal dysfunction for pharmacotherapy in SCI}

For proper pharmacotherapy after SCI, early renal dysfunction detection is critical. Renal excretion of intact drugs and their metabolites is carried out by a combination of three processes: glomerular filtration, TS and tubular reabsorption. Glomerular elimination of drugs depends on molecular size and protein binding. Although protein binding decreases filtration of drugs, it may increase the amount that renal tubules secrete.

Our results suggest that renal drug handling pathways are not affected equally after SCI: GFR is altered while TS remains unchanged. Under these circumstances, clearance of drugs that are eliminated primarily by GFR will be decreased and their plasma half-life prolonged, as we observed here.

Optimal pharmacotherapy management of cord-injured subjects requires knowledge of pharmacological characteristics of drugs, as well as patients' physiological alterations. Effectiveness and safety of pharmacological treatments are important to ensure a good quality of life and of the rehabilitation process. Impaired renal function compels adjustments of drug dosage, in particular those excreted by glomerular filtration, which are potentially toxic, such as aminoglycosides.

Further investigation in relation to SCI and renal drug handling should include development of simple and reliable methods to identify early renal dysfunction in the clinical setting, as well as to design practical dosing guidelines as a function of renal performance. Undoubtedly, the greatest challenge is to reverse the pathophysiological mechanisms that cause renal dysfunction in the first place. Apparently, the main mechanism involved is GFR decrease observed here during the acute stage of SCI is ischemia.

\section{CONCLUSIONS}

Acute SCI causes renal dysfunction characterized by decreased GFR (as measured by iohexol clearance), but preserved TS (as determined by $\mathrm{PAH}$ clearance screening). Our results contribute to the 
understanding of fundamental aspects of renal elimination of drugs after SCI during spinal shock.

Alterations of GFR result in decreased drug clearance and consequently increased drug bioavailability and increased risk of drug toxicity.

Professionals who care for patients in the early stages after cord injury should find this information useful when administering drugs eliminated by the kidney.

\section{DATA ARCHIVING}

There were no data to deposit.

\section{CONFLICT OF INTEREST}

The authors declare no conflict of interest.

1 Guízar-Sahagún G, Castañeda-Hernández G, García-López P, Franco-Bourland R, Grijalva I, Madrazo G. Pathophysiological mechanisms involved in systemic and metabolic alterations secondary to spinal cord injury. Proc West Pharmacol Soc 1998; 41: 237-240.

2 Segal JL, Pathak MS. Optimal drug therapy and therapeutic drug monitoring after spinal cord injury: a population-specific approach. Am J Ther 2001; 8: 451-463.

3 Cruz-Antonio L, Arauz J, Franco-Bourland RE, Guízar-Sahagún G, Castañeda-Hernández G. Contrasting effects of cord injury on intravenous and oral pharmacokinetics of diclofenac: a drug with intermediate hepatic extraction. Spinal Cord 2012; 50: 632-635.

4 García-López P, Martínez-Cruz A, Guízar-Sahagún G, Castañeda-Hernández G. Acute spinal cord injury changes the disposition of some, but not all drugs given intravenously. Spinal Cord 2007; 45: 603-608.

5 Mohler LJ, Ellison FM, Flanigan CR. Creatinine clearance prediction in spinal cord injury patients: comparison of 6 prediction equations. J Urol 1988; 139. 706-709.

6 Waldman J, Eisenberg B, Murata HG, Anderson X. Comparison of nomogram- and ultrasound-determined renal depths for calculation of glomerular filtration rates in spinal cord injury patients. Clin Nucl Med 1992; 17: 958-960.

7 Stein DM, Menaker J, McQuillan K, Handley C, Aarabi B, Scalea TM. Risk factors for organ dysfunction and failure in patients with acute traumatic cervical spinal cord injury. Neurocrit Care 2010; 13: 29-39.
8 Huerta MO, González FL, Treviño BA, Meza-Coria CC, Castañeda-Hernández G. Development and validation of an analytical method for the determination of serum concentration of iohexol, a marker of glomerular filtration. Rev Mex Cienc Farm 2012; 43: 46-53.

9 Savant IA, Kalis M, Almoazen H, Ortiz SR, AbuTarif M, Taft DR. Alternative highperformance liquid chromatographic assay for p-aminohippuric acid (PAH): effect of aging on PAH excretion in the isolated perfused rat kidney. J Pharm Biomed Anal 2001; 26: 687-699.

10 Guízar-Sahagún G, Velasco-Hernández L, Martínez-Cruz A, Castañeda-Hernández G, Bravo G, Rojas $\mathrm{G}$ et al. Systemic microcirculation after complete high and low thoracic spinal cord section in rats. J Neurotrauma 2004; 21: 1614-1623.

11 Bravo G, Guízar-Sahagún G, Ibarra A, Centurion D, Villalón CM. Cardiovascular alterations after spinal cord injury: an overview. Current Med Chem Cardiovasc Hematol Agents 2004; 2: 133-148.

12 Lehmann KG, Lane JG, Piepmeier JM, Batsfors WP. Cardiovascular abnormalities accompanying acute spinal cord injury in humans: incidence, time course and severity. J Am Coll Cardiol 1987; 10: 46-52.

13 Gondim FA, Lopes Jr AC, Oliveira GR, Rodrigues CL, Leal PR, Santos AA et al. Cardiovascular control after spinal cord injury. Curr Vasc Pharmacol 2004; 2: 71-79.

14 Popa C, Popa F, Grigorean VT, Onose G, Sandu AM, Popescu M et al. Vascular dysfunctions following spinal cord injury. J Med Life 2010; 3: 275-285.

15 Gris D, Hamilton EF, Weaver LC. The systemic inflammatory response after spinal cord injury damages lungs and kidneys. Exp Neurol 2008; 211: 259-270.

16 Young $\mathrm{F}$, Ensom MH. Pharmacokinetics of aminoglycosides in patients with chronic spinal cord injury. Am J Health Syst Pharm 2011; 68: 1607-1614.

17 MacDiarmid SA, Mclntyre WJ, Anthony A, Bailey RR, Turner JG, Arnold EP. Monitoring of renal function in patients with spinal cord injury. BJU Int 2000; 85: 1014-1018.

18 Shihabi ZK, Constantinescu MS. Iohexol in serum determined by capillary electrophoresis. Clin Chem 1992; 38: 2117-2120.

19 Gaspari F, Perico N, Matalone M, Signorini O, Azzollini N, Mister M et al. Precision of plasma clearance of iohexol for estimation of GFR in patients with renal disease. J Am Soc Nephrol 1998; 9: 310-313.

20 Brown SC, O'Reilly PH. Iohexol clearance for the determination of glomerular filtration rate in clinical practice: evidence for a new gold standard. J Urol 1991; 146: 675-679.

21 Sturgeon C, Sam II AD, Law WR. Rapid determination of glomerular filtration rate by single-bolus inulin: a comparison of estimation analyses. J App/ Physiol 1998; 84: 2154-2162.

22 Reyes LJ, Meléndez E, Alegría A, Jaramillo-Juárez F. Influence of sex differences on the renal secretion of organic anions. Endocrinology 1998; 139: 1581-1587.

23 Haberle D, Ober A, Ruhland G. Influence of glomerular filtration rate on the rate of para-aminohippurate secretion by the rat kidney: micropuncture and clearance studies. Kidney Int 1975; 7: 385-396. 